\title{
Pigmented villonodular synovitis of the knee in a patient on oral anticoagulation therapy: a case report Balasundaram Ramesh*, Sanathkumar Shetty and Salah S Bastawrous
}

Address: Department of Trauma and Orthopaedics, Glan Clwyd Hospital, Sarn Lane, Bodelwyddan, Rhyl, North Wales, LL18 5UJ, UK

Email: Balasundaram Ramesh* - orthoygc@yahoo.com; Sanathkumar Shetty - sanatusha@yahoo.com;

Salah S Bastawrous - Salah.Bastawrous@cd-tr.wales.nhs.uk

* Corresponding author

Published: 13 November 2009

Journal of Medical Case Reports 2009, 3:121 doi:10.1186/1752-1947-3-121
Received: 7 January 2008

Accepted: 13 November 2009

This article is available from: http://www.jmedicalcasereports.com/content/3/I/I2I

(C) 2009 Ramesh et al; licensee BioMed Central Ltd.

This is an Open Access article distributed under the terms of the Creative Commons Attribution License (http://creativecommons.org/licenses/by/2.0), which permits unrestricted use, distribution, and reproduction in any medium, provided the original work is properly cited.

\begin{abstract}
Introduction: Pigmented villonodular synovitis is a disease which affects the synovial joints and tendon sheaths. Although the exact aetiological factors are not known, we believe that recurrent haemarthrosis has a role in the aetiology of this condition.

Case presentation: A 62-year-old Caucasian man presented with gradually worsening pain and stiffness in his right knee. The patient was on anticoagulation therapy and had been treated for recurrent episodes of spontaneous haemarthrosis of the knee. The International Normalized Ratio on each occasion suggested poor control of the anticoagulation therapy. A diagnosis of pigmented villonodular synovitis was made based on intra-operative findings and was further confirmed by a histopathological examination.
\end{abstract}

Conclusion: This report is presented to highlight the unusual association of haemarthrosis and pigmented villonodular synovitis.

\section{Introduction}

Pigmented villonodular synovitis (PVNS) is a disease of unknown aetiology affecting the synovial joints. The aetiology of PVNS remains controversial and a number of theories have been postulated. Haemarthrosis has been suggested as a possible aetiological factor. Only one description of PVNS of the ankle in a patient on anticoagulation therapy has been previously reported [1]; we describe the second known case of PVNS of the knee joint in a patient on anticoagulation therapy.

\section{Case presentation}

A 62-year-old Caucasian man had an uneventful total left knee replacement five years prior to presentation. Two years following the total knee replacement, the patient was diagnosed with dilated cardiomyopathy and was started on warfarin. Following this, he had recurrent episodes of sudden pain and swelling on his right knee. During each of these episodes, there was no history of trauma and the patient was systemically well. Although his International Normalized Ratio (INR) was high, his blood tests for full blood count and C-reactive protein were within the normal range. All of these episodes were in the initial phase of his warfarin therapy and they ceased once his INR was stabilized.

The condition of the patient's right knee gradually deteriorated. Clinically, the knee joint was diffusely swollen and tender but stable. His active range of movement was from neutral to 100 degrees of flexion and any further flexion was painful. Radiographs of the knee showed advanced arthritic changes and he was admitted for a total 
knee replacement. Intraoperatively, the synovium was found to be hypertrophic and stained reddish orange and the synovial fluid was reddish-orange in colour (Figure 1). These appearances suggested a diagnosis of PVNS. A synovectomy was performed, which was then followed by a total knee replacement. A synovial specimen was sent for histopathological examination. The microscopic features were consistent with a diagnosis of PVNS (Figure 2). The postoperative period was uneventful and the patient was asymptomatic after three years of follow-up treatment.

\section{Discussion}

PVNS typically occurs in adults in their third or fourth decade of life, with a male-to-female ratio of 1.9 to 1.3. Involvement is usually monoarticular [2]. The knee joint is the most frequently affected site, followed by the fingers, feet, ankles, hips, wrists and shoulders in a decreasing order of frequency $[2,3]$.

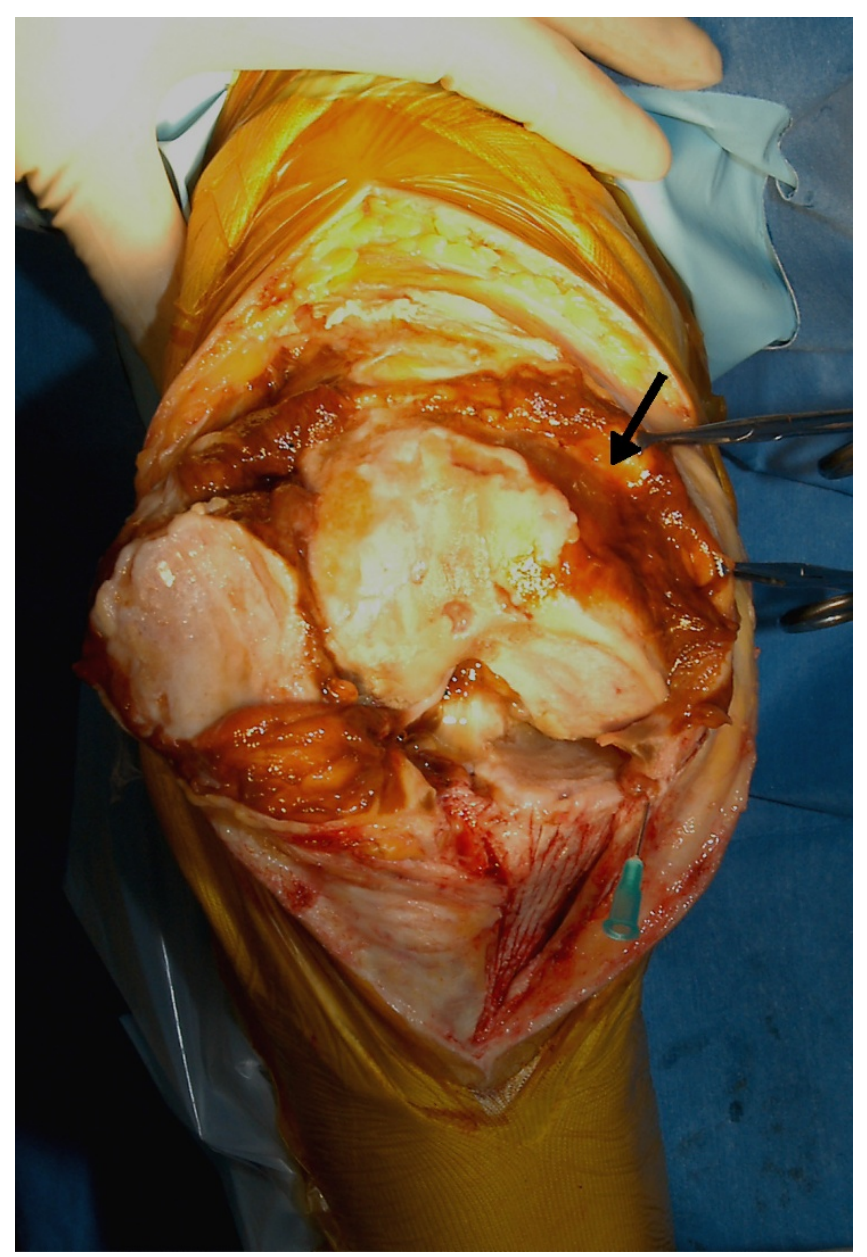

Figure I

Intra-operative photographs showing the reddishorange stained synovium (black arrow).

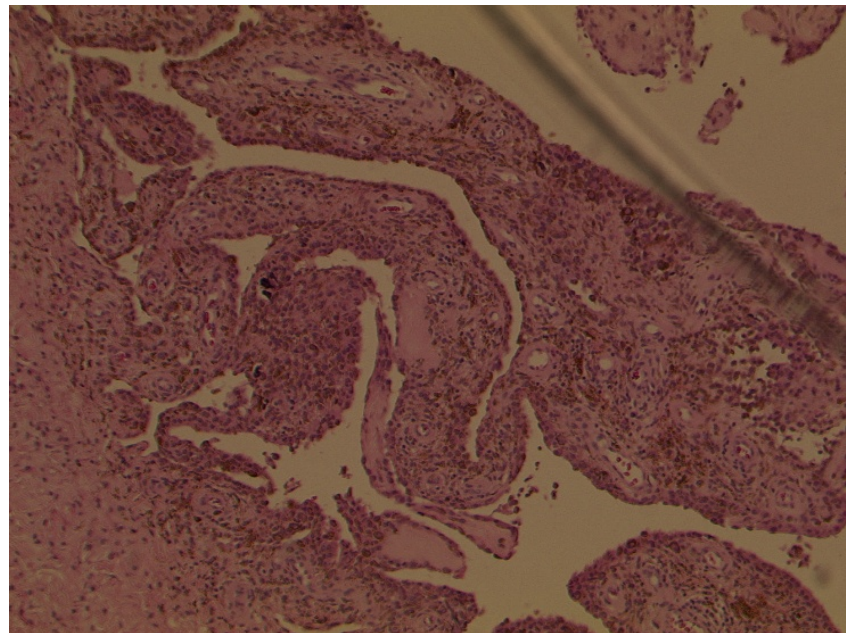

Figure 2

Photomicrograph showing the presence of haemosiderin deposits, foamy histiocytes and giant cells confirming pigmented villonodular synovitis (haematoxylin and eosin stain).

Since the first description of this condition by Jaffe et al. in 1941 [4], the aetiology of this benign tumour involving the synovial membrane has remained unclear. Jaffe proposed that a hypervascular cellular phase occurs after trauma produc ing hyalinization and fibrosis [4]. Various aetiologies including trauma [2], inflammation [3], haemorrhage [5], neoplasia [6] and genetic factors [7] have been suggested. Chronic recurrent microtrauma and haemarthrosis have also been postulated [2].

It has also been postulated that PVNS in children arises through a different mechanism to that in adults, and it is also possible that not all lesions interpreted as PVNS share the same mechanism [8].

There are very few cases of PVNS reported in patients on anticoagulation therapy [1] and with a bleeding disorder [9]. In our patient, the symptoms in the knee worsened following these repeated episodes of haemarthrosis and the INR on these occasions showed a poor control of his anticoagulation therapy.

\section{Conclusion}

This case supports the argument of earlier reports [1,9] that repeated haemarthrosis may have a role in the aetiology of PVNS. We hope that this study will encourage the reporting of similar cases to lead to a better understanding of the aetiology of this condition.

\section{Abbreviations}

INR: International Normalized Ratio; PVNS: pigmented villonodular synovitis. 


\section{Competing interests}

The authors declare that they have no competing interests.

\section{Authors' contributions}

SS made substantial contributions in acquiring data, reviewing the literature and preparing the manuscript. BR performed the knee replacement operation and also contributed in reviewing the literature and drafting the manuscript. SSB gave final approval to the draft to be published. All authors read and approved the final manuscript.

\section{Consent}

Written informed consent was obtained from the patient for publication of this case report and any accompanying images. A copy of the written consent is available for review by the Editor-in-Chief of this journal.

\section{References}

I. Pearse OE, Klass B, Bendall SP: Pigmented villonodular synovitis of the ankle occurring in a patient on anticoagulation therapy. J Surg Orthop Adv 2004, I 3(4):217-219.

2. Myers BW, Masi AT: Pigmented villonodular synovitis and tenosynovitis: a clinical epidemiologic study of 166 cases and literature review. Medicine 1980, 19:223-238.

3. Granowitz SP, O'Antorio J, Mankin $\mathrm{H}$ : The pathogenesis and long term end results of pigmented villonodular synovitis. Clin Orthop Relat Res 1976, I 1 4:335-35I.

4. Jaffe HL, Lichtenstein L, Sutro C]: Pigmented villonodular synovitis, bursitis and tenosynovitis. Arch Pathol 194I, 3 I:73 I-765.

5. Leszczynski J, Huckell JR, Percy JS, LeRiche JC, Lentle BC: Pigmented villonodular synovitis in multiple joints. Occurrence in a child with cavernous hemangioma of lip and pulmonary stenosis. Ann Rheum Dis 1975, 34:269-272.

6. Rao AS, Vigorite VJ: Pigmented villonodular synovitis (giant cell tumor of tendon sheath and synovial membrane). Review of 8 I cases. J Bone Joint Surg Am 1984, 66:76-94.

7. Wendt RG, Wolfe F, McQueen D, Murphy P, Solomon H, Housholder $\mathrm{M}$ : Polyarticular pigmented villonodular synovitis in children: evidence for genetic contribution. J Rheumatol I986, I3(5):921-926.

8. Schumacher HR, Lotke P, Athreya B, Rothfuss S: Pigmented villonodular synovitis: light and electron microscopic studies. Semin Arthritis Rheumatol 1982, I 29(I):32-43.

9. Matsui H, Takahashi Y, Matsunaga T, Tanaka-Horie T, Minowa H, Sugimoto M, Tsukino R, Mii Y, Giddings J, Yoshioka A: Successful arthroscopic treatment of pigmented villonodular synovitis of the knee in a patient with congenital deficiency of plasminogen activator inhibitor-I and recurrent haemarthrosis. Haemostasis 2001, 31:106-II2. 